\title{
Numerical Solution of Steady-State Free Boundary Problems using the Singular Boundary Method
}

\author{
Fen Chen ${ }^{1,2}$, Bin Zheng ${ }^{3, *}$, Ji Lin ${ }^{3}$ and Wen Chen ${ }^{3}$ \\ 1 College of Civil Engineering, Hefei University of Technology, Hefei, Anhui 230009, \\ China \\ ${ }^{2}$ College of Civil Engineering, Taizhou University, Taizhou, Zhejiang 318000, China \\ ${ }^{3}$ College of Mechanics and Materials, Hohai University, Nanjing, Jiangsu 211100, \\ China
}

Received 24 June 2019; Accepted (in revised version) 17 September 2019

\begin{abstract}
In this paper, the recently-developed singular boundary method is applied to address free boundary problems. This mesh-less numerical method is based on the use of the origin intensity factors with fundamental solutions. Three numerical examples and their results are compared with the results obtained using traditional methods. The comparisons indicate that the proposed scheme yields good results in determining the position of the free boundary.
\end{abstract}

AMS subject classifications: 00A69

Key words: Seepage flow, singular boundary method, mesh-less, origin intensity factors.

\section{Introduction}

Water seepage is the leading cause of a considerable number of geological disasters. The determination of the free boundary for the seepage plays a major part in this problem. This problem has been extensively studied by variable grid methods, fixed grid methods, and mesh-less methods in recent decades.

Liao proposed the residual pressure feedback method [1] for the simulation of free surface flow. Neumann put forward the Galerkin method [2] which was continuously optimized and developed as an element-free method [3]. Subsequently, a large variety of grid methods attempted to detect the location of free boundary, such as the residual flow method [4], the osmosis matrix adjustment [5], the initial flow method [6], the variational inequality [7], and the finite element method (FEM) [8]. The disadvantages of these methods are that they are time-consuming and inflexible.

*Corresponding author.

Email: zhengbinyilan@163.com (B. Zheng) 
Based on a set of scattered nodes, mesh-less methods overcome certain limitations of grid methods. The Kansa method [9], the boundary knot method (BKM) [10], the backward substitution method [11,12], and the method of fundamental solutions (MFS) [1315] are such methods. Nevertheless, the MFS shows superiority of stability over the LRBF.

Chen and his collaborators devised a new boundary-type mesh-less method, namely the singular boundary method (SBM), which is a coupling between the MFS and the indirect boundary element method (BEM) [16]. The SBM employs the fundamental solutions as the basis functions, and introduces the concept of origin intensity factors $[17,18]$ to take place of the singularities encountered in the fundamental solutions at origin. The method inherits the dimensional superiority of the BEM and does not involve costly integration. The method eliminates the complicated fictitious boundary issue associated with the traditional MFS. Based on the SBM, highly accurate results have been obtained in various wave cases [19-21], large-scale problems [22], transient matters [23,24], and heat conduction problems in layered materials $[25,26]$. In this paper, the SBM is extended to solve steady-state free boundary problems.

The rest of the paper is organized as follows. In Section 2, we give a brief review on the basic idea of the SBM for solving steady-state free boundary problems. In Section 3 , in order to demonstrate the effectiveness of the SBM, three numerical examples are presented. A summary and conclusions are provided in the last section.

\section{The SBM for the free boundary problems}

We consider the following problem [27]

$$
\nabla^{2} \phi(\mathbf{x})=0, \quad \mathbf{x} \in \Omega,
$$

subject to the boundary conditions:

$$
\begin{aligned}
& B \phi(\mathbf{x})=f, \quad \mathbf{x} \in \partial \Omega_{\mathrm{FIXED}}, \\
& \left\{\begin{array}{l}
B_{1} \phi(\mathbf{x})=f_{1}, \\
B_{2} \phi(\mathbf{x})=f_{2},
\end{array} \quad \mathbf{x} \in \partial \Omega_{\mathrm{FREE}},\right.
\end{aligned}
$$

where $\partial \Omega=\partial \Omega_{\mathrm{FIXED}} \cup \partial \Omega_{\mathrm{FREE}}, \partial \Omega_{\mathrm{FIXED}}$ is the fixed boundary and $\partial \Omega_{\mathrm{FREE}}$ is the free boundary. $B$ denotes the boundary operator on the fixed boundary. The two different kinds of boundary operators on the free boundary are denoted by $B_{1}$ and $B_{2} . f, f_{1}$ and $f_{2}$ represent known functions. The least squares fit to the data generated is treated as follows :

$$
F=\left\{\sum_{m=1}^{M_{\mathrm{FIXED}}}\left|B \phi\left(\mathbf{x}_{m}\right)-f\right|^{2}+\sum_{m=1}^{M_{\mathrm{FREE}}}\left[\left|B_{1} \phi\left(\mathbf{x}_{m}\right)-f_{1}\right|^{2}+\left|B_{2} \phi\left(\mathbf{x}_{m}\right)-f_{2}\right|^{2}\right]\right\}^{\frac{1}{2}} .
$$

$\phi(\mathbf{x})$ is an approximate solution with unknown coefficients. $M_{\text {FIXED }}$ and $M_{\text {FREE }}$ denote the number of fixed boundary and free boundary nodes, respectively. In this paper, the 


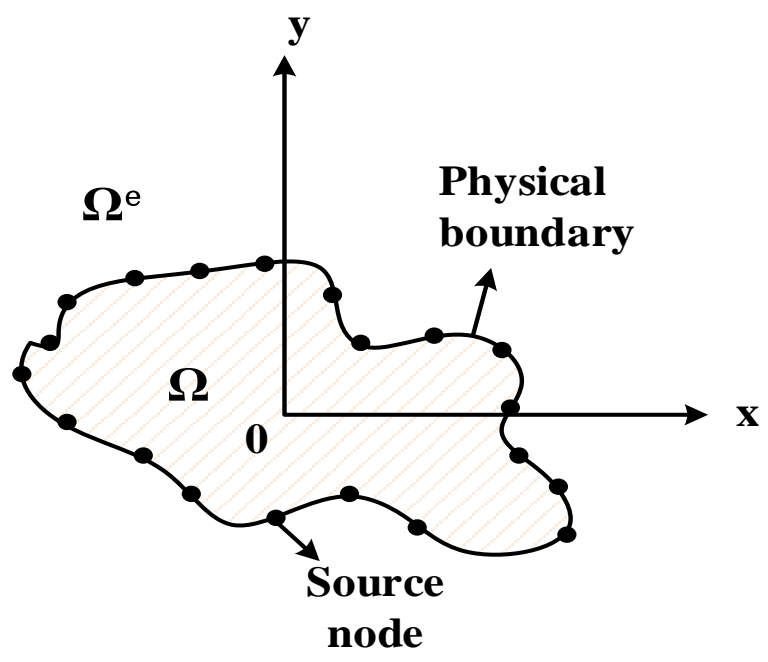

Figure 1: Source nodes on the physical boundary.

$y$-coordinates of the free boundary nodes could move vertically while the $x$-coordinates remain fixed.

The unit normal vector $\mathbf{n}$ of a point on a curve $y=g(\mathbf{x})$ is defined by

$$
\mathbf{n}=\frac{\left( \pm g^{\prime}(\mathbf{x}), \pm 1\right)}{\sqrt{1+g^{\prime}(\mathbf{x})^{2}}}
$$

We approximated $g^{\prime}\left(\mathbf{x}_{m}\right)$ with a central difference approximation:

$$
g^{\prime}\left(\mathbf{x}_{m_{1}}\right)=\frac{\mathbf{x}_{m+1_{2}}-\mathbf{x}_{m-1_{2}}}{\mathbf{x}_{m+1_{1}}-\mathbf{x}_{m-1_{1}}}, \quad m=2, \cdots, M-1,
$$

where $M$ denotes the number of boundary nodes. The unit normal vectors of the edge points can be determined by forward and backward finite difference approximations. To illustrate the SBM, we consider Eq. (2.1) under the following boundary conditions:

$$
\begin{array}{ll}
\phi(\mathbf{x})=\bar{\phi}(\mathbf{x}), & \mathbf{x} \in \Gamma_{D}, \\
\nabla \phi \cdot \mathbf{n}=q(\mathbf{x}), & \mathbf{x} \in \Gamma_{N},
\end{array}
$$

where $\partial \Omega=\Gamma_{D} \cup \Gamma_{N}$. The first step to solve the boundary value problem by the SBM is to arrange the source nodes (Fig. 1) on the physical boundary of the computational domain. 
After the source nodes are determined, the SBM takes the follow approximation

$$
\begin{array}{lll}
\bar{\phi}\left(\mathbf{x}_{m}\right)=\sum_{n=1}^{N} \alpha_{n} G_{L}\left(\mathbf{x}_{m}, \mathbf{s}_{n}\right)+c, & \mathbf{x}_{m} \in \Omega, \\
q\left(\mathbf{x}_{m}\right)=\sum_{n=1}^{N} \alpha_{n} \frac{\partial G_{L}\left(\mathbf{x}_{m}, \mathbf{s}_{n}\right)}{\partial \mathbf{n}_{\mathbf{x}_{m}}}, & & \mathbf{x}_{m} \in \Omega,
\end{array}
$$

where

$$
G_{L}(\mathbf{x}, \mathbf{s})=-\frac{1}{2 \pi} \ln \left\|\mathbf{x}_{m}-\mathbf{s}_{n}\right\|
$$

is the fundamental solution of the two-dimensional Laplace equation. The $\mathbf{S}_{n}$ denote the source nodes and the $\mathbf{X}_{m}$ represent the collocation nodes in the region. The MFS arranges the source nodes on a fictitious boundary outside the physical region to avoid the coincidence of the source nodes and the collocation nodes. The SBM places the source nodes and collocation nodes which are the same set of nodes on the boundary of the physical domain. $N$ represents the number of the nodes. The $\left\{\alpha_{n}\right\}$ are coefficients to be determined and $c$ is an unknown constant to ensure uniqueness of the solution. Meanwhile, the coefficients should be consistent with following supplementary condition to solve the constant $c$,

$$
\sum_{n=1}^{N} \alpha_{n}=0
$$

which is also called as the moment condition.

The concept of origin intensity factor is introduced to replace the singular term when the source nodes coincide with collocation nodes

$$
\begin{aligned}
& \phi\left(\mathbf{s}_{m}\right)=\sum_{n \neq m}^{N} \alpha_{n} G_{L}\left(\mathbf{s}_{m}, \mathbf{s}_{n}\right)+\alpha_{m} \widetilde{G}_{L}\left(\mathbf{s}_{m}, \mathbf{s}_{m}\right)+c, \\
& q\left(\mathbf{s}_{m}\right)=\sum_{n \neq m}^{N} \alpha_{n} \frac{\partial G_{L}\left(\mathbf{s}_{m}, \mathbf{s}_{n}\right)}{\partial \mathbf{n}_{\mathbf{s}_{m}}}+\alpha_{m} \widetilde{Q}_{L}\left(\mathbf{s}_{m}, \mathbf{s}_{m}\right),
\end{aligned}
$$

where $\widetilde{G}_{L}\left(\mathbf{s}_{m}, \mathbf{s}_{m}\right)$ and $\widetilde{Q}_{L}\left(\mathbf{s}_{m}, \mathbf{s}_{m}\right)$ denote the origin intensity factors.

For the natural boundary (Neumann) conditions (Fig. 2), the origin intensity factors [28] are

$$
\widetilde{Q}_{L}\left(\mathbf{s}_{m}, \mathbf{s}_{m}\right)=-\sum_{n \neq m}^{N} \frac{L_{n}}{L_{m}} \frac{\partial G_{L}\left(\mathbf{s}_{m}, \mathbf{s}_{n}\right)}{\partial \mathbf{n}_{\mathbf{s}_{n}}},
$$

where $L_{n}$ is the half arc length between sources $\mathbf{s}_{n-1}$ and $\mathbf{s}_{n+1}$ (Fig. 3). The empirical formula [29] on the Dirichlet boundary is

$$
\widetilde{G}_{L}\left(\mathbf{s}_{m}, \mathbf{s}_{m}\right)=-\frac{1}{2 \pi} \ln \left(\frac{L_{m}}{2 \pi}\right) .
$$




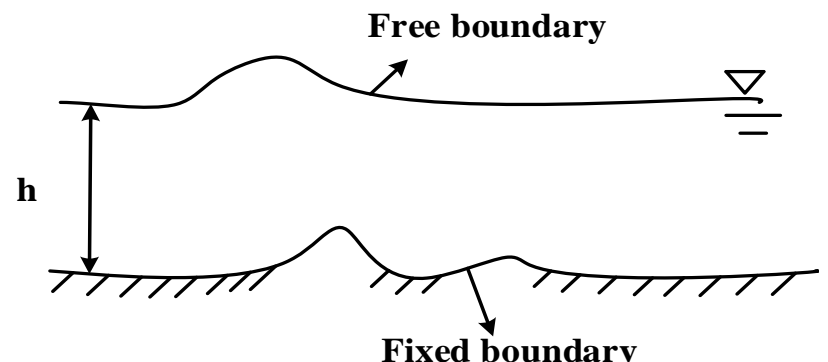

Fixed boundary

Figure 2: Investigated problem.

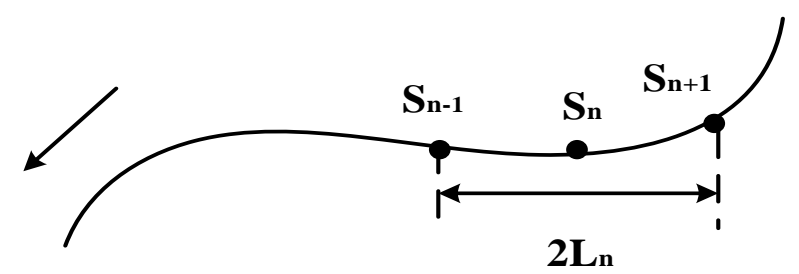

Figure 3: The length of $\mathbf{L}_{n}$ for 2D problem.

According to Eqs. (2.12)-(2.13), the following standard linear algebraic system can be obtained:

$$
\left\{\begin{array}{ccc}
\widetilde{G}_{L}\left(s_{m}, s_{m}\right) & G_{L}\left(s_{m}, s_{n}\right) & 1 \\
\widetilde{Q}_{L}\left(s_{m}, s_{m}\right) & \frac{\partial G_{L}\left(s_{m}, s_{n}\right)}{\partial n_{s_{m}}} & 0 \\
1 & 1 & 0
\end{array}\right\}\left\{\alpha_{N+1}\right\}=\left\{\begin{array}{c}
\phi\left(s_{m}\right) \\
q\left(s_{m}\right) \\
0
\end{array}\right\} .
$$

The matrix form is

$$
\mathbf{A} \alpha=\mathbf{b},
$$

where $\mathbf{A}$ is the coefficient matrix, $\{\alpha\}$ represent the vectors of unknown coefficients and b denote the right-hand side vectors. The unknown vectors $\{\alpha\}$ can be determined by solving this system.

Tips: Step-by-step computational procedure is displayed as follows:

Step 1 Assume initial coordinates of the free boundary nodes and discretize the fixed boundary.

Step 2 Solve Eqs. (2.1)-(2.2b) using the SBM. 
Step 3 Substituting the original coordinates into the Eqs. (2.9a)-(2.9b), new $\Phi\left(s_{m}\right)$ or $q\left(s_{m}\right)$, which include the updated coordinates of free boundary nodes are obtained. Substituting the updated coordinates into the Eq. (2.3), if the value does not reach the tolerance, average the original and updated coordinates to obtain a set of trial coordinates. Don't stop substituting the trial coordinates into the Eqs. (2.9a)-(2.9b) until the value by the Eq. (2.3) reaches the tolerance.

Step 4 Determine the free boundary by fitting curves with the new coordinates of the nodes.

\section{Numerical results and discussions}

Example 3.1 (Seepage through a rectangle porous dam). In the first example, we apply the SBM to solve seepage flow through a rectangular mass soil, which is depicted in Fig. 4 [30].

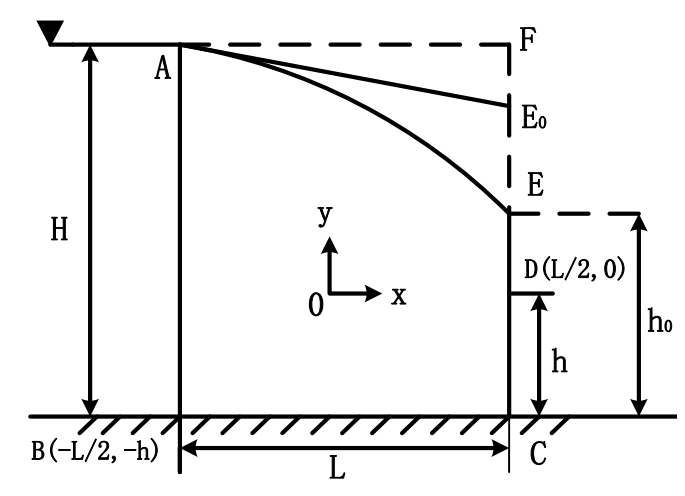

Figure 4: Seepage through a rectangle porous dam.

The seepage $(A E)$ is a dividing line which is the free boundary between wet and dry soil. The governing equation for the potential function $\varphi(x, y)$ is

$$
\nabla^{2} \varphi=0 \text { in } A B C D E A,
$$

subject to the boundary conditions

$$
\begin{array}{ll}
\varphi=H-h & \text { on } A B \\
\frac{\partial \varphi}{\partial \mathbf{n}}=0 & \text { on } B C, \\
\varphi=0 & \text { on } C D, \\
\varphi=y & \text { on } D E
\end{array}
$$




$$
\left\{\begin{array}{l}
\varphi=y, \\
\frac{\partial \varphi}{\partial \mathbf{n}}=0,
\end{array} \quad \text { on } A E .\right.
$$

The potential function $\varphi(x, y)$, shape of the curve $A E$ and $h_{0}(C E)$ are unknown, where $L=10, h=3$ and $H=10 . A E_{0}$ is an initial assumed line and $h<C E_{0}<H$. Furthermore, the $x$-coordinates of free boundary nodes remain fixed while the $y$-coordinates can move vertically. To obtain the coordinates of the edge node, we use linear interpolation with nodes which are close to the point $E$. The results by the MFS with an adjustable fictitious boundary in [30], the MFS with a fixed fictitious boundary and the SBM are presented in Table 1. It is not reasonable to compare the computational cost of the MFS [30] with other methods because the results were obtained over two decades ago. The fictitious boundary of the MFS is set an appropriate distance from the physical boundary by multiple error analysis. The shape of the fictitious boundary is similar to the physical boundary. The source nodes are evenly distributed on the fictitious boundary. The height $h_{0}$ obtained by the SBM in Table 1 is in good agreement with the analytical solution as shown in Polubarinova-Kochina [31] $\left(h_{0} \approx 4.20\right)$.

In Table $1, M$ and $M_{\text {FREE }}$ represent the number of boundary nodes and free boundary nodes, respectively. NFEV denotes the number of function evaluations. $N$ is the number of iterations. The computational time is shown in seconds. Note that the iteration of the SBM for this example is much less than the MFS. Furthermore, it takes less CPU time by the SBM. The shapes of $A E$ obtained by the SBM are shown in Fig. 5.

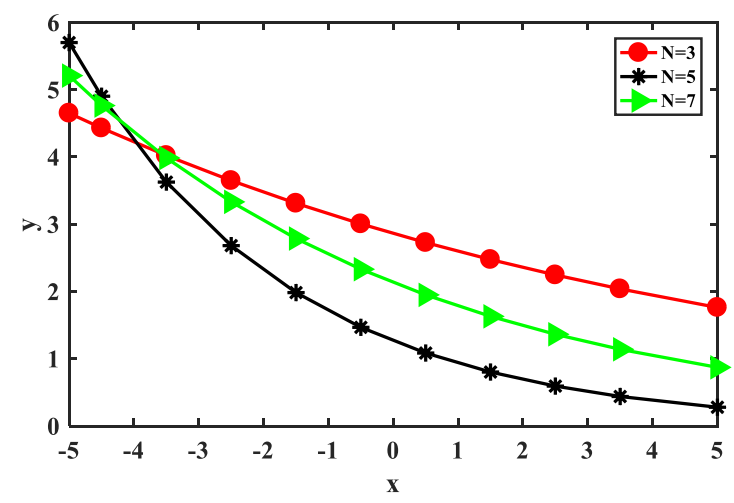

Figure 5: Location of free boundary by the SBM.

Example 3.2 (Cavity flow). As shown in Fig. 6, an incompressible inviscid fluid passes a plate with finite width and infinite length [30]. What we need is to use image plate to exploit the shape of cavity. Due to symmetry, we can only consider a quarter of region displayed in Fig. 7. 
Table 1: Results of the Example 3.1.

\begin{tabular}{||cc|ccc|ccc||}
\hline \multicolumn{7}{||c|}{$M=68, M_{\text {FREE }}=10$} \\
\hline \multicolumn{2}{|c|}{ MFS [30] } & \multicolumn{3}{c||}{ MFS } & \multicolumn{3}{c||}{ SBM } \\
\hline NFEV & $h_{0}$ & $N$ & $h_{0}$ & CPU(s) & $N$ & $h_{0}$ & CPU(s) \\
\hline 600 & 4.147 & 200 & 4.299 & 0.30 & 3 & 5.154 & 0.02 \\
800 & 4.212 & 300 & 4.276 & 0.42 & 4 & 5.228 & 0.03 \\
1000 & 4.223 & 400 & 4.275 & 0.53 & 5 & 4.176 & 0.03 \\
& & & & & 6 & 4.212 & 0.03 \\
& & & & & 7 & 4.226 & 0.03 \\
\hline
\end{tabular}

The governing equation for stream function in region ABCDEFA is as follows:

$$
\nabla^{2} \Psi=0 \text { in } A B C D E F A,
$$

subject to the following boundary conditions

$$
\begin{array}{ll}
\frac{\partial \Psi}{\partial \mathbf{n}}=0 & \text { on } C D, A B, \\
\Psi=0 & \text { on } D E, E F, \\
\Psi=1 & \text { on } B C, \\
\begin{cases}\Psi=0, & \text { on } F A . \\
\frac{\partial \Psi}{\partial \mathbf{n}}=-q, & \end{cases}
\end{array}
$$

The free boundary $F A$ is a major part of the shape of the cavity. According to [30], we take $L=0.5, C D=1(h=1.0), D E=1.5$ and $d=0.1$. $F A$ is assumed as a straight line initially. To determine the location of $F A$, the $x$-coordinates of nodes remain constant while the $y$-coordinates are allowed to move vertically. In addition, the $y$-coordinates of the nodes on $A B$ are re-adjusted. The constant $q$ is set as zero originally. The edge point is obtained by interpolating the nodes near the point $F$. We analyse sufficient errors to identify an appropriate distance between the fictitious boundary and the physical boundary of the MFS. The source nodes are selected on the fictitious boundary evenly. The fictitious boundary is taken to be similar to the physical boundary. The constant $q$ and value $b$ are consistent with results obtained in Aitchison [33] by the FEM and Aitchison and Karageorghis [34] with the boundary integral equation method. The results obtained by the MFS with adjustable fictitious boundary in [30], the MFS with fixed fictitious boundary and the SBM are listed in Table 2.

$M$ denotes the number of boundary nodes and $M_{\mathrm{FREE}}$ represents the number of free boundary nodes. NFEV is the number of function evaluations. $N$ is the number of iterations and CPU shows the computational time. The locations of the free boundary (FA) obtained by the MFS with fixed fictitious boundary and the SBM are shown in Fig. 8. 


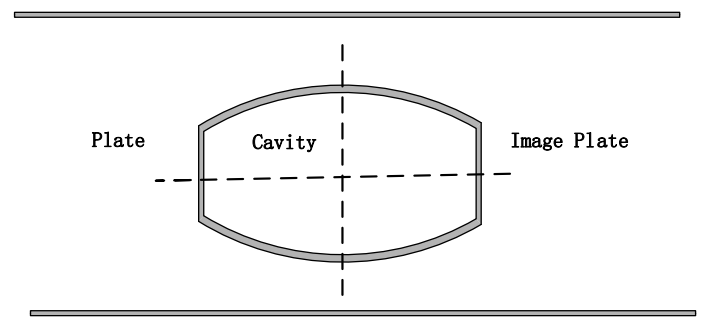

Figure 6: Cavity model.

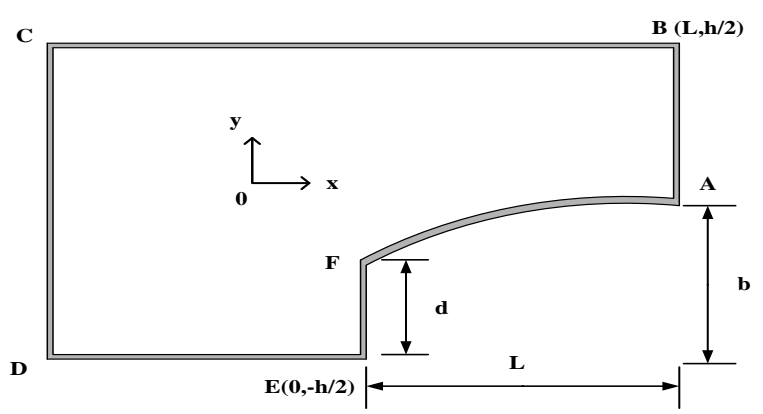

Figure 7: Solution region.

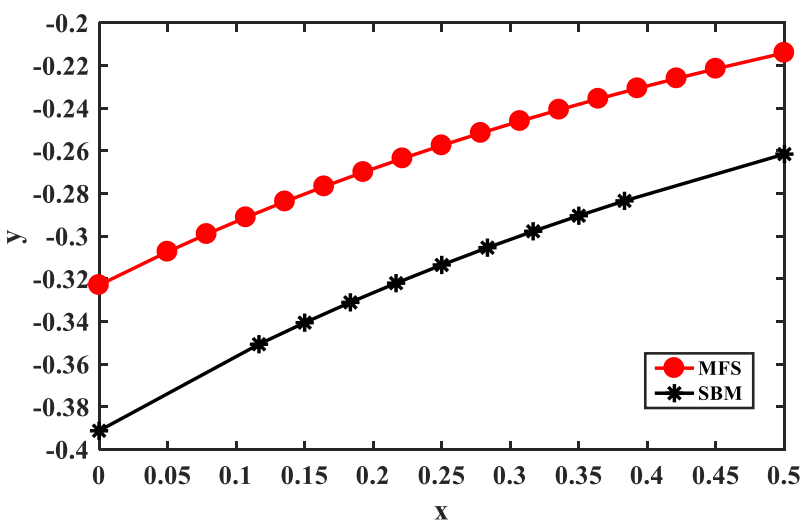

Figure 8: Location of free boundary by the MFS and SBM.

Example 3.3 (Flow over a triangular weir). In Example 3.3, we examine potential flow over a triangular weir under gravity [30]. Line GF is the free boundary (Fig. 9). Stream 
Table 2: Results of the Example 3.2.

\begin{tabular}{|c|c|c|c|c|c|c|c|c|c|c|}
\hline \multicolumn{7}{|c|}{$M=132, M_{\text {FREE }}=17$} & \multicolumn{4}{|c|}{$M=132, M_{\text {FREE }}=11$} \\
\hline \multicolumn{3}{|c|}{ MFS [30] } & \multicolumn{4}{|c|}{ MFS } & & & & \\
\hline$q$ & $b$ & NFEV & $q$ & $b$ & $N$ & CPU(s) & $q$ & $b$ & $N$ & CPU(s) \\
\hline 1.4491 & 0.2102 & 1000 & 1.4491 & 0.2827 & 1000 & 1.44 & 1.4491 & 0.2159 & 1000 & 8.77 \\
\hline Aitchison [33] & & & 1.429 & 0.2013 & & & & & & \\
\hline $\begin{array}{c}\text { Aitchison and } \\
\text { Karageorghis [34] }\end{array}$ & & & 1.4753 & 0.2222 & & & & & & \\
\hline
\end{tabular}

Table 3: Results of Example 3.3.

\begin{tabular}{||cc|cc||}
\hline \multicolumn{2}{||c||}{$M=197, M_{\text {FREE }}=45$} & \multicolumn{2}{c||}{$M=197, M_{\text {FREE }}=9$} \\
\hline \multicolumn{2}{||c|}{ MFS [30] } & \multicolumn{2}{c||}{ SBM } \\
\hline 885 & 3.3440 & 496 & $q$ \\
965 & 3.3409 & 586 & 3.2524 \\
1045 & 3.3393 & 676 & 3.33025 \\
\hline
\end{tabular}

function $\Psi(x, y)$ is displayed below.

$$
\nabla^{2} \Psi=0 \text { in } A B C D E F G A,
$$

subject to boundary conditions

$$
\begin{aligned}
& \nabla^{2} \Psi=0 \quad \text { on } A B C D E \text {, } \\
& \frac{\partial \Psi}{\partial \mathbf{n}}=0 \quad \text { on } E F, G A \text {, } \\
& \left\{\begin{array}{l}
\Psi=q, \\
\frac{\partial \Psi}{\partial \mathbf{n}}=\sqrt{2 g\left(H_{0}-y-0.5\right)}, \quad \text { on } F G .
\end{array}\right.
\end{aligned}
$$

According to Aitchison [32], EF $\left(h_{2}\right)$ and $G A\left(h_{1}\right)$ satisfy the following cubic equation

$$
z^{3}-H_{0} z^{2}+\frac{q^{2}}{2 g}=0
$$

The equation above has two real positive roots $z_{1}$ and $z_{2}$ in $\left[0, H_{0}\right]$ (neglect the negative one), $z_{1} \in\left[0,2 H_{0} / 3\right]$ and $z_{2} \in\left[2 H_{0} / 3, H_{0}\right]$. The physically interesting flows are defined by

$$
\begin{array}{ll}
h_{1}=h_{2}=z_{1} & \text { (supercritical flow), } \\
h_{1}=h_{2}=z_{2} & \text { (subcritical flow), } \\
h_{1}=z_{2}, \quad h_{2}=z_{1} & \text { (critical flow). }
\end{array}
$$

The critical flow is a case where $H_{0}$ or $q$ is given. In [32], $H_{0}=1.138, X R=X L=3.0$ and $p=0.2$. The coefficient $q$ is taken as one initially. GF is set to be a straight line at the 


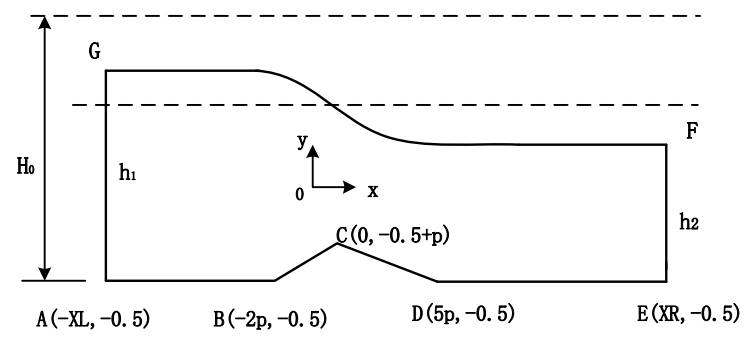

Figure 9: Flow over a triangular weir.

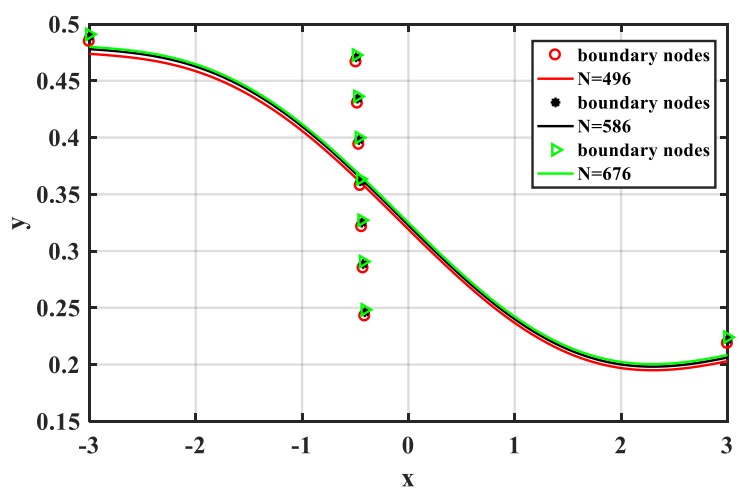

Figure 10: Location of free boundary by the SBM.

beginning. The Results, listed in Table 3, obtained by the SBM converge to 3.30 that is in good agreement with the MFS in [30].

The number of boundary nodes is $M$. $M_{\text {FREE }}$ denotes the number of free boundary nodes. NFEV represents the number of functional evaluations. $N$ shows the number of iterations. Locations of the free boundary $(G F)$ obtained by the SBM are displayed in Fig. 10.

\section{Conclusions}

In this paper, the SBM is extended to solve free boundary problems. The method is simple, easy in implementation and highly accurate. It avoids singularities through the use of the origin intensity factors with fundamental solutions. Numerical results validate the accuracy and effectiveness of the SBM in solving free boundary problems. It should be noted that a full matrix is formed when the SBM is employed. Hence, some fast solvers will be used if there is plenty of boundary nodes. Application to the transient free boundary problems by the SBM will be investigated in the future. 


\section{Acknowledgements}

We would like to thank the editor and referees for giving valuable improvements to the paper. The work was supported by the National Natural Science Foundation of China (No. 11702083) and the open research fund of Guangxi key laboratory of water engineering materials and structures, Guangxi institute of water resources research (No. GXHRIWEMS-2019-05).

\section{References}

[1] H. S. LIAO, W. L. XU AND C. G. WU, Residual pressure feedback method for simulation of free surface flow, J. Hydraulic Eng., 124 (1998), pp. 1049-1052.

[2] S. P. NeumAnN, Saturated-unsaturated seepage by finite elements, J. Hydraulics Division-Asce, 99 (1973), pp. 2233-2250.

[3] X. L. LI, Error estimates for the moving least-square approximation and the element-free Galerkin method in n-dimensional spaces, Appl. Numer. Math., 99 (2016), pp. 77-97.

[4] C. S. DESAI, Finite element residual scheme for unconfined flow, Int. J. Numer. Methods Eng., 10 (1979), pp. 1415-1418.

[5] K. J. BATHE AND M. R. KHOSHGOFTAAR, Finite element free surface seepage analysis without mesh iteration, Int. J. Numer. Anal. Methods Geomech., 3 (2010), pp. 13-22.

[6] Y. ZHANG, P. CHEN AND L. WANG, Initial flow method for seepage analysis with free surface, J. Hydraulic Eng., 8 (1988), pp. 18-26.

[7] T. DESPERAT, Free boundary problems in the theory of fluid flow through porous media, Annali Di Matematica Pura Ed Applicata, 97 (1973), pp. 1-82.

[8] B. Y. SU AND Y. M. ZHU, Node virtual discharge method for seepage problems with free surface on fixed grids, Journal of Hohai University Natural Science, 5 (1991), pp. 113-117.

[9] E. J. KANSA, Multiquadrics-A scattered data approximation scheme with applications to computational fluid-dynamics-II solutions to parabolic, hyperbolic and elliptic partial differential equations, Comput. Math. Appl., 19 (1990), pp. 147-161.

[10] W. CHEN, Symmetric boundary knot method, Eng. Anal. Boundary Elements, 26 (2002), pp. 489-494.

[11] J. LIN, S. Y. REUTSKIY AND JUN LU, A novel meshless method for fully nonlinear advectiondiffusion-reaction problems to model transfer in anisotropic media, Appl. Math. Comput., 339 (2018), pp. 459-476.

[12] J. LIN, Y. XU, AND Y. H. ZHANG, Simulation of linear and nonlinear advection-diffusion-reaction problems by a novel localized scheme, Appl. Math. Lett., 99 (2020), 106005.

[13] J. LIN, C. S. CHEN, C. S. LIU, AND J. LU, Fast simulation of multi-dimensional wave problems by the sparse scheme of the method of fundamental solutions, Comput. Math. Appl., 72(3) (2016), pp. 555-567.

[14] A. Poullikkas, A. Karageorghis, G. Georgiou And J. Ascough, The method of fundamental solutions for Stokes flows with a free surface, Numer. Methods Partial Differential Equations, 14 (1998), pp. 667-678.

[15] A. KARAGEORGHIS AND G. FAIRWEATHER, The method of fundamental solutions for axisymmetric potential problems, Int. J. Numer. Methods Eng., 44 (2015), pp. 1653-1669.

[16] G. Q. WANG AND F. L. GUO, A stochastic boundary element method for piezoelectric problems, Eng. Anal. Boundary Elements, 95 (2018), pp. 248-254. 
[17] J. P. LI, W. CHEN AND Y. GU, Error bounds of singular boundary method for potential problems, Numer. Methods Partial Differential Equations, 33 (2017), pp. 1987-2004.

[18] L. L. SUN, W. CHEN AND H. D. CHENG, Evaluating the origin intensity factor in the singular boundary method for three-dimensional Dirichlet problems, Adv. Appl. Math. Mech., 9 (2017), pp. 1289-1311.

[19] Z. J. FU, W. CHEN, P. H. WEN AND C. Z. ZHANG, Singular boundary method for wave propagation analysis in periodic structures, J. Sound Vibration, 425 (2018), pp. 170-188.

[20] W.W. LI AND W. CHEN, Band gap calculations of photonic crystals by singular boundary method, J. Comput. Appl. Math., 315 (2017), pp. 273-286.

[21] J. Lin, C. Z. ZHANG, L. L. SUN AND J. LU, Simulation of seismic wave scattering by embedded cavities in an elastic half-plane using the novel singular boundary method, Adv. Appl. Math. Mech., 10 (2018), pp. 322-342.

[22] W. W. LI, W. ChEN AND G. F. PANG, Precorrected-FFT accelerated singular boundary method for large-scale three-dimensional potential problems, Commun. Comput. Phys., 22 (2017), pp. 460-472.

[23] F. J. WANG, W. Chen, A. TAdeu And C. G. Correia, Singular boundary method for transient convection-diffusion problems with time-dependent fundamental solution, Int. J. Heat Mass Transfer, 114 (2017), pp. 1126-1134.

[24] X. WEI, AND L. SUN, Singular boundary method for 3D time-harmonic electromagnetic scattering problems, Appl. Math. Model., 76 (2019), pp. 617-631.

[25] B. ZHENG, J. LIN AND W. CHEN, Simulation of heat conduction problems in layered materials using the meshless singular boundary method, Eng. Anal. Boundary Elements, 100 (2019), pp. $88-94$.

[26] X. WeI, W. Chen, B. Chen, AND L. Sun, Singular boundary method for heat conduction problems with certain spatially varying conductivity, Comput. Math. Appl., 69 (2015), pp. $206-$ 222.

[27] R. MATHON AND R. L. JOHNSTON, The Approximation solution of elliptic boundary-value problems by fundamental solutions, SIAM J. Numer. Anal., 14 (1977), pp. 638-650.

[28] Y. GU AND W. CHEN, Infinite domain potential problems by a new formulation of singular boundary method, Appl. Math. Model., 37 (2013), pp. 1638-1651.

[29] J. P. LI, W. CHEN AND Z. J. FU, Explicit empirical formula evaluating origin intensity factors of singular boundary method for potential and Helmholtz problems, Eng. Anal. Boundary Elements, 73 (2016), pp. 161-169.

[30] A KARAGEORGHIS, The method of fundamental solutions for the solution of steady-state free boundary problems, J. Comput. Phys., 98 (1992), pp. 119-128.

[31] P. Ya Polubarinova-Koch And J. M. Roger De Wiest, Theory of Ground Water Movement, Princeton University Press, New Jersey, USA, 2015.

[32] J. M. AitCHISON, A variable finite element method for the calculations of flow over a weir, Rutherford Laboratory 1979-Annual Report, No. RL-79-069, 1979.

[33] J. M. AITCHISON, The numerical solution of planar and axisymmetric cavitational flow problems, Comput. Fluids, 12 (1984), pp. 55-65.

[34] J. M. AITCHISON, AND A KARAGEORGHIS, Numerical solution of a free surface problem by a boundary element method, Int. J. Numer. Methods Fluids, 8 (1988), pp. 91-96. 